\title{
Managing configurable products in the computer industry: Planning and coordination issues
}

\author{
RAMESH SRINIVASAN ${ }^{1}$ and JAYASHANKAR M SWAMINATHAN ${ }^{2}$ \\ ${ }^{1}$ IBM TJ Watson Research Center, Yorktown Heights, NY 10598, USA \\ ${ }^{2}$ Walter A Haas School of Business, University of California, Berkeley, CA \\ 94720 , USA \\ e-mail: sramesh@watson.ibm.com; msj@haas.berkeley.edu
}

\begin{abstract}
With the increase in the drive towards greater customer satisfaction, there has been a proliferation in the variety of products offered by manufacturers. Variety provides considerable choice to customers and encourages manufacturers to modularize their product lines. However, associated with a broad product line are complexities related to design, forecasting, coordination and operations. In this paper, we discuss some of these important issues.
\end{abstract}

Keywords. Product variety; feature-based product lines; operations management; forecasting; planning; computer industry.

\section{Introduction}

In a global economy, manufacturers are driven towards offering broader product lines as they have to cater to the needs of customers with very different requirements. This has led manufacturers to offer customizable products in different configurations utilizing common building blocks also known as feature-based product lines. Although offering more variety in the product line increases the number of market segments that are covered (Bagozzi 1986), it also increases the complexity of the manufacturing process, thereby increasing operational costs (Abeggelen \& Stalk 1985). Increase in variety also increases the number of components and sub-assemblies that are utilized. For example, General Motors Corporation offered 131 different rear-axle assemblies with the intent of providing variety in pickup trucks (Fonte 1994). Such increases in variety lead to greater complexities in forecasting, parts planning, final assembly and delivery of products.

In a feature-based product line, each product is defined in terms of configurations which are built from a feature-set offered by the manufacturer. This provides customers with the ability to have their choice of features while defining the product configuration. In addition, intense competition between manufacturers has made the time to deliver, or responsiveness, an important factor in determining their success. As a result, customers have more choice in terms of product features and have greater control over lead times that they can expect 
once orders are placed. On the other hand, manufacturers face an uphill task because while keeping costs under control they have to deal with uncertainty in customer demands and respond quickly once customer demands are known. Since the number of configurations demanded by customers is enormous, there are additional challenges to the manufacturer in demand forecasting and production planning.

In order to overcome these challenges, manufacturers are adopting several new techniques. These include incorporation of operational constraints during design of the product, delaying differentiation of products during assembly, integrating information within the organization and across the supply chain, clustering products into product families, exploiting commonality in components and manufacturing processes and using better decision-support tools for forecasting and parts planning. For example, Ford Motors has started designing cars for a global market while keeping in mind the various options that it may need to provide to different customer zones (Treece et al 1995). Hewlett Packard and IBM are delaying product differentiation in order to provide variety in their product line while keeping costs under control (Swaminathan \& Tayur 1996). Toyota has been clustering products into families to exploit commonality in order to develop cost effective designs (Gupta \& Krishnan 1995). While these techniques have shown considerable success in particular cases where they were employed, in a broader perspective it is essential to understand challenges that may arise when variety in the product line is increased.

In this paper our primary focus is on the computer industry and we highlight some of the important issues related to forecasting, parts planning, final assembly and distribution that arise as a result of increase in product variety. The rest of the paper is as follows. In $\S 2$, we describe feature-based product structure in greater detail with an example. In $\S 3$, we describe issues related to forecasting. In $\$ 4$, we describe some of the issues related to parts planning, final assembly and interplant coordination and in $\$ 5$ we briefly describe other issues related to managing feature-based product lines and provide our concluding remarks.

\section{Feature-based product lines}

In this section, we introduce the concept of a feature-based product line through an example from the personal computer industry. Traditionally, in the personal computer industry manufacturers have been offering predefined computer models to customers. Over time as a response to customer requirements the number of models grew enormously. For example, at the beginning of this decade IBM offered a few hundred models of personal computer. Such large numbers of predefined end-products resulted in complexities in forecasting and planning and inefficiencies in operations. This led computer manufacturers to experiment with new product offering strategies. One such strategy is to offer a featurebased product line. As opposed to offering numerous predefined models to customers, a feature-based product line permits customers to define their choice of configuration based on hardware and software features provided by the manufacturer. In general, a feature is a subassembly or subsystem to which a function can be ascribed from a customer perspective. Examples of features are: a graphics hardware kit, token-ring card, $1 \mathrm{~GB}$ direct-access storage device etc. 

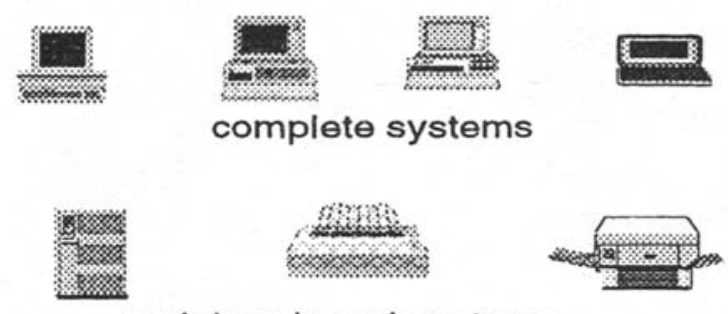

peripherals, subsystems

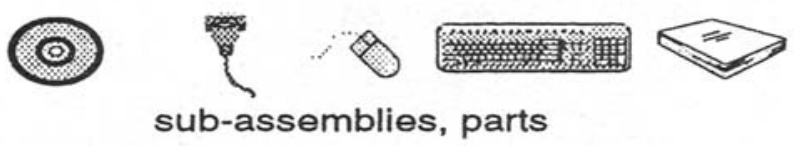

Figure 1. Customer-orderable items.

A customer's order can be made up of a collection of entities drawn from system units, subsystems, peripheral, other features and parts (see figure 1). A system unit is defined by a customer's choice of feature categories such as processor, hard-drive, memory, power-supply, communication and multi-media features (refer to figure 2). Certain rules are observed in the specification of a system unit. For example, a customer may not be allowed to choose a low speed processor and a very high capacity hard-drive. A featurebased approach provides adequate flexibility in defining a system unit. For example, a customer may not buy a hard-drive with a system unit, but may have it installed separately. Some features like power supply and processor are mandatory in defining a system unit while others like memory are optional. In each feature category, a customer makes a specific choice from the list of options provided. For example, there are hundreds of options available to choose to satisfy communications and multi-media capability requirements.

Each option in every feature category has a unique bill-of-materials graph as shown in figure 3. For example, part $A$ is an assembly that is made up of 1 unit of part J, 2 units of part K and 1 unit of part L. Similar to a conventional bill-of-materials (BOM), the BOM for a feature-based product line has the characteristics of substitution among parts and effectivity windows for parts, two important factors that add complexity to planning. A substitute part is one which can be used when the primary part is unavailable. There may be more than one substitute part for a primary part and these may be primary parts in a

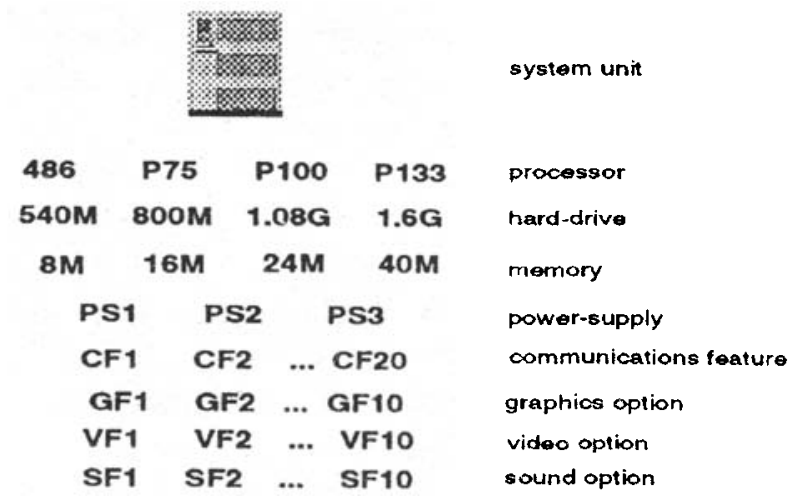

Figure 2. Flexible product structure. 


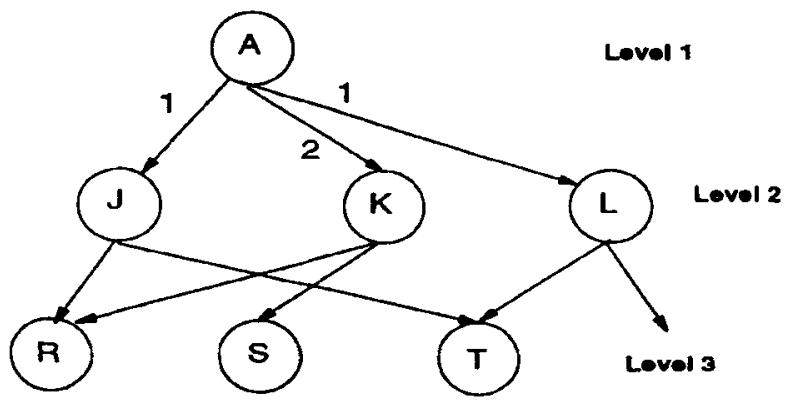

Figure 3. Bill-of-materials.

different section of the bill-of-materials structure. An effectivity window associated with a part specifies the time window during which this part can be used in manufacturing. Outside this time window, other parts specified in the bill-of-materials definition should be used. An effectivity window (defined later) for a part is usually introduced due to considerations related to engineering changes and change of supplier.

Feature-based product offering also exists in mid-range and main-frame product lines. In such product lines, a major source of demand for computer hardware and software is the customer's need to upgrade to higher capacity or the latest technology. The demand for upgrades can be greater than fifty percent and as high as ninety percent. This results in the need to offer subsystems and other modular enhancements to existing systems.

\section{Implications for requirements forecasting}

Plenty of research has been done on forecasting of production and inventory requirements for manufacturing industries. A good review of forecasting techniques can be found in Fildes \& Beard (1992). The authors point out that serious gaps exist in the knowledge necessary to design an effective forecasting system for a production enterprise. Two key points are made in this regard. First, an effective approach to forecast demand for a product has to utilize information specific to the product. Secondly, information on which a forecast is based should include data in addition to time-series history, such as orders, marketing plans and product life-cycle. We find these conclusions to be very appropriate in the case of feature-based products.

Increased variety in product offering has important effects on product development, product upgrade possibilities and customer orders. In the following subsections, these effects and their consequences on demand forecasting are described.

\subsection{Product transition}

The product development function in a corporation has the responsibility to develop profitable products that closely match customer requirements. This is done based on market research and leads to strategies for introduction of new products and withdrawal of old products, often referred to as product transition. The function and form of new products as well as the timing of introduction are some of the decisions made and conveyed to 
the forecasting and business planning units. Since the development of a product involves coordination and completion of hundreds of activities, the actual product content realized and the month or quarter of a year when a product becomes available for volume production are subject to a high degree of uncertainty. Increase in product variety makes each product development project very complex, thereby aggravating the difficulty in estimating the resulting product content and time of volume production. This results in inaccuracy of forecast for existing products since time and extent of cannibalization of their demand by new products are subject to uncertainty. In addition, sustaining existing products beyond previously planned months or quarters could require new parts suppliers or an increase in plant capacity, which may be difficult to manage.

These difficulties resulting from uncertainties in product development can be handled by quantitative models. The product development project should be explicitly represented as a stochastic process made up of several parallel and sequential activities. On analyzing such a process, different possible completion dates for the product development activity and their probabilities can be derived. Several demand forecasts for the new product and existing product can then be predicated by these completion dates.

\subsection{Increased upgrading possibilities}

As indicated earlier, a significant portion of demand for mid-range and main-frame systems arises from the customer's need to upgrade existing hardware capacity or upgrade to new technology. This results in demand for subsystems, e.g. storage systems and processors, in addition to what may be demanded with the main computer system. Upgrading also implies the need for compatibility of subsystems with existing hardware. This often results in additional parts which constitute an upgrade kit. The accuracy of forecasting demand for upgrades can benefit from approaches that consider current installed base of computers in the market place and the upgrading possibilities for the installed base of computers. In case of high-end or main-frame systems, where the volume is low, projection of demand for upgrades could be performed by considering each existing customer individually and identifying upgrade possibilities depending on the customer's current product configuration. Since there may be several upgrade possibilities for each customer, a probabilistic model of upgrade possibilities is appropriate. When the customer population is large, as in mid-range computers and high-end workstations, the customer base should be segmented according to existing configurations before projecting demand. It is to be noted that even in a traditional product offering, customers could upgrade their hardware. However, such an upgrade is usually from one predefined end-product to another. As a result the complexities in forecasting mentioned above are not prevalent.

\subsection{Customer requirements}

Traditionally, a few models were offered to end-customers and forecasters could focus on projecting demand for each of them. Each model represented an adequately differentiated functionality and as a result, segmentation of customer population by functionality resulted in disjoint sets. However, in the case of feature-based product lines the offering is described as feasible collections of modular and interchangeable subsystems and subassemblies, 
referred to as common building blocks (figure 2). With the usage of building-blocks in product offerings, segmentation of customer population by functionality is not precise. At the same time the number of final product configurations is too huge to develop individual forecasts with high accuracy. This has led to an alternate strategy in firms such as IBM PC Company where forecasts for individual building-blocks are generated instead of forecasts for customer demanded configurations. This process is well suited for features such as microprocessors and disk-drives which are included in most customer orders. For example, in the personal computer industry forecasters use the demand for individual commodities such as Intel's 486 processors and 4 M DRAM chips. Both end-product forecasting and building-block forecasting have their own advantages. The advantages of end-product forecasting are the following:

(1) It eases mapping to end-customer population segmentation;

(2) Characterization of demand dependencies among subsystems and sub-assemblies is easier;

(3) It eases the financial planning and monitoring at assembly plants who perform their calculations at the end-product level;

(4) It facilitates predicting customer service at the end-product level for a pre-specified safety stock allocation.

On the other hand, building-block forecasting has its own advantages which are the following:

(1) The forecasts at the building-block level are more reliable because they are aggregated across all the products;

(2) Industry level forecasts are easily available for commodities or building-blocks.

Approaches that combine the advantages of end-product forecasting and commodity forecasting do not exist and need to be developed. This is particularly essential in businesses which sell whole-systems, subsystems, sub-assemblies and parts.

\section{Operational challenges}

An increase in product variety and customization leads to a proliferation of components and sub-assemblies as well as increases complexity in the manufacturing process. Empirical research indicates that the degree to which the above factors influence costs, depends on the manufacturing process and techniques utilized. For example, Kekre \& Srinivasan (1990) and Fisher et al (1993) indicate that broader product lines do not increase manufacturing cost significantly. On the other hand, MacDuffie et al (1996) and Banker et al (1990) find that product variety and complexity have a significant impact on operational costs. Kekre \& Srinivasan (1990) attribute the difference to adoption of better manufacturing techniques including set-up reduction, just-in-time manufacturing, increasing commonality in the product line and providing operational flexibility.

In order to make their operations competitive, manufacturers need to pay closer attention to issues related to parts planning, final assembly and distribution. We highlight some of these issues in the following subsections. 


\subsection{Parts planning}

Parts planning deals with developing detailed production and procurement plans for all the components and sub-assemblies that go into the final product. The parts-planning problem in a conventional product line, where products are pre-defined by components has been addressed by a number of researchers in academia and industry. When demands for endproducts are deterministic, the problem of determining which products and how many of such products to build given constraints on component supply has been formulated as a linear program (Dietrich et al 1995). When demands are stochastic, the problem has been addressed by formulating it as a stochastic program and solving it by different techniques. Exploiting the commonality of components across assemblies through risk-pooling is an important feature in the stochastic problem. Industrial size problems have been addressed by the solution approach described by (Srinivasan et al 1992), where heuristic solutions for the stochastic problem have been proposed.

In the case of feature-based products, it will not be known a priori which configurations the customers will order. The number of feasible configurations could be too huge for demand to be specified for each configuration separately. This issue exists in the case of deterministic demands as well as stochastic demands. In the deterministic case the demand may be specified by a single number, total volume, for the entire product line. In addition, a ratio will be specified for each feature in the product line; the demand for the feature is then obtained by multiplying the total volume by the ratio. For example, one might have information that a hard disk of type $A$ is used in $60 \%$ of orders for machine type 1111 . This information in itself is not adequate because a hard disk of type A may be ordered with a higher likelihood if the product configuration has a processor of type X. Since such a specification does not explicitly show any interdependencies which may exist among features, this demand representation is incomplete. Consequently, additional assumptions are needed to determine parts requirements. Approaches to perform parts planning for feature-based product lines in deterministic and siochastic contexts are currently being pursued at IBM Research.

\subsection{Assembly planning}

Manufacturers face a tough challenge in providing a large variety to customers under conditions where demand is uncertain and a quick response is needed once it is known. Some of the leading manufacturers in the computer industry including Hewlett-Packard and IBM are trying to delay differentiation of the final product. Delayed differentiation (also called postponement) involves storing inventory in semi-finished forms and finishing the product configuration by adding components once demand is known. For example, HP decided to store inventory in the form of partially configured printers and add power supply and documentation at local distribution centres in Europe. This led to a great deal of reduction in inventory costs mainly due to risk pooling effects (Lee \& Billington 1993).

In the case of IBM, product differentiation was delayed by storing semi-finished inventory also called vanilla boxes. The term vanilla (white) represented the fact that they were not final (coloured) products and box is a terminology used for a finished product. When 
customer orders for final configurations were known with certainty, additional components were added to the vanilla box and the product was shipped to the customer. An additional constraint here was that the product had to be delivered to the customer within a certain time limit from the time the order was placed. In such a situation, it was a challenging task to determine (a) how to allocate the assembly capacity between assembling vanilla boxes and final products; (b) how many types of vanilla boxes to keep (how many different partial configurations), what features to include in them and how much inventory to build each period; (c) how to allocate the existing vanilla boxes to satisfy current demand for final products. Swaminathan \& Tayur (1996) provide an algorithm that can solve industry size problem within reasonable time. Their study shows that vanilla assembly process performs very well when product demands are negatively correlated and there is a high degree of commonality in the product line. Delaying differentiation at the final assembly stage helps in improving the performance of the manufacturer as compared to both assemble-to-order (all the assembly operations are done only after demand is known) and make-to-stock environments under the above conditions.

An important concem while adopting delayed differentiation is related to the correlation between demands for final products as well as the correlation between the demand for features. It seems that an ideal vanilla box is made up of features that are positively correlated in order to support a set of products whose demands are negatively correlated. Another important concern while trying to adopt such a strategy relates to capacity available at the final assembly stage. If additional capacity can be acquired at reasonable cost, then it may be optimal to operate under an assemble-to-order environment. A make-to-stock environment is preferable if the number of end-configurations is limited and demands between the products are independent or positively correlated. Swaminathan \& Tayur (1996) analyse only a single level of BOM for end configurations. The more detailed problem involves consideration of features and options within each of these features. Research is in progress to address such problems.

The effectiveness of providing product variety depends to a great extent on the manufacturing process. One of the prime concerns here relates to set-up reduction. If the set-up for changing from one type to another type of the product is low then one could effectively manage production of a variety of parts or products. For example, Whitney (1993) describes how set-up reduction through better design improved the effectiveness of operations at Nippon Denso while offering more types of radiators.

\subsection{Inter-plant coordination}

Coordination between different plants of a supply chain is a challenging task. Outsourcing leads to complications in terms of coordination with suppliers as discussed in the section on parts planning. In addition, production and distribution of parts, sub-assemblies and products has many interesting aspects. Cohen \& Lee (1988), Cohen \& Moon (1990), Newhart et al (1993) have discussed supply chain coordination issues under deterministic scenarios and a global optimization criterion. Lee \& Billington (1993) and Pyke \& Cohen $(1993,1994)$ consider stochastic environments and provide approximations to optimal inventory levels, reorder intervals and service levels. Arntzen et al (1995) develop an elaborate model for global supply chain management for Digital Equipment Corporation. 
Most of the above work relates to product structures where the bill of material is fixed and known in advance. As a result, final assembly, packing peripherals and adding documentation can be coordinated in advance based on the type of product. However, in a feature-based product line, it is a complicated task to plan the merger of the subsystems comprising the order with other peripherals and documentation since the contents of the orders are not known a priori. In addition, it is more difficult to synchronize the arrival of the product and peripherals at the customer site since additions to the product may come from other manufacturing units. Manufacturers like IBM have primarily considered two alternatives. In the first alternative the product and peripherals are merged in transit which implies that they are synchronously received at the receiving dock. Alternatively, they are brought separately to a consolidation centre where they are merged. Consolidation centres generally have a cross-docking operation where safety stocks are not stored. In the first alternative, the challenge lies in effective transfer of information between various units in the supply chain so that the order can be completed in transit. In the second alternative, the challenge lies in effective coordination of receipt of parts at the consolidation centres so that the amount of time spent in the consolidation centre is minimized. It is to be noted that the primary difference between the two alternatives is the absence of a physical location for consolidation in the first case. For a large organization, there are also concerns regarding whether to run the consolidation centres on their own or get all the distribution services from an external vendor.

In addition to consolidating the product, there are also issues related to how products should be delivered from distribution centres to retailers. Issues related to where inventory should be stored and who should bear the financial burden for it are also very important (Anupindi \& Bassok 1995). As is evident from the above subsections, operational challenges faced by a manufacturer are tougher while managing a broader product line. We have only highlighted some of the issues and have neglected those related to the shop floor such as process control, line-balancing, scheduling and batching. These issues are equally important and provide their share of difficulties while managing a broader product line.

\section{Conclusions}

In this paper we first introduce the notion of a feature-based product line through an example from the computer industry. Subsequently we have highlighted important challenges that manufacturers have to face in the areas of planning and operations management. In particular, we discussed issues related to forecasting, product transition, upgrading products, parts planning, final assembly and interplant coordination. We also mention some of the important problems that manufacturers face for which accurate solution methodologies are not available today.

Our focus in this paper was primarily on planning and coordination issues. However, there are other issues related to offering a broad product line some of which are mentioned below:

- Pricing decisions are extremely important for managing any product line because that in most part determines the demand generated for products. Pricing decisions are difficult 
in a feature based product line because of the difficulty in segmenting the market based on functionality.

- Coordination between marketing and manufacturing in order to decide on the right set and number of features to be promoted in the product line.

- Modularity and commonality in the product line offer additional challenges while trying to integrate operations aspects in the early part of the design because one has to consider the impact of changes in design of a part on the whole product line rather than a single product. This could lead to changes in design specifications for even external suppliers.

- In high-technology industries there is an additional challenge related to choosing the right design for modules because the product life-cycles are short and a design that could be compatible with more than one generation of products may benefit from economies of scale.

- Number and location of components and final assembly plants and distribution centres need to be carefully determined because of increase in product variety and customization of different products.

Increased product variety benefits customers, while at the same time helping manufacturers to adequately differentiate their products. However, in order to successfully offer product variety a manufacturer may have to effectively overcome many of the challenges identified in this paper.

The authors thank the referees and the Guest Editor for comments and suggestions that have improved the quality of the paper.

\section{References}

Abeggelen J C, Stalk G 1985 KAISHA, The Japanese Corporation (New York: Basic Books)

Anupindi R, Bassok Y 1995 Centralization of stocks: Retailers vs. manufacturer. Working Paper, Northwestern University

Arntzen B G, Brown G G, Harrison T P, Trafton L L 1995 Global supply chain management at Digital Equipment Corporation. Interfaces 25: 69-93

Bagozzi R P 1986 Principles of marketing management (Chicago, IL: Science Research Associates)

Cohen M A, Lee H L 1988 Strategic analysis of integrated production distribution systems. $J$. Oper. Res. 36: 216-228

Cohen M A, Moon S 1990 Impact of production scale economies, manufacturing complexity and transportation costs on supply chain facility networks. J. Manuf. Oper. Manage. 3: 269-292

Banker R D, Datar S M, Kekre S, Mukhopadhyay T 1990 Costs of product and process complexity. In Measures of manufacturing excellence (ed.) R Kaplan (Boston: Harvard Business School Press)

Dietrich B, Connors D, Ervolina T, Fasano J P, Lin G, Srinivasan R, Wittrock R, Jayaraman R 1995 Production and procurement planning under resource availability constraints and demand variability. IBM Research Report, RC 19948 
Fildes R, Beard C 1992 Forecasting systems for production and inventory control. Int. J. Oper. Production Manage. 12: 4-27

Fisher M L, Jain A, MacDuffe J P 1993 Strategies for product variety: Lessons from the auto industry. Working Paper, Wharton School, University of Pennsylvania

Fonte W G 1994 A de-proliferation methodology for the automotive industry. Master's thesis, Massachusetts Institute of Technology, Leaders of Manufacturing Program

Gupta S, Krishnan V 1995 Product family-based assembly sequence design to advance the responsiveness-customization frontier. Working Paper, University of Texas, Austin

Kekre S, Srinivasan K 1990 Broader product line: A necessity to achieve success? Manage. Sci. 36: $1216-1231$

Lee H L, Billington C 1993 Material management in decentralized supply chains. J. Oper. Res. 41: 835-847

MacDuffie J P, Sethuraman K, Fisher M L 1996 Product variety and manufacturing performance: Evidence from the international automotive assembly plant study. Manage. Sci. 42: 350-369

Newhart D D, Scott K L, Vasko F J 1993 Consolidating product sizes to minimize inventory levels for a multi-stage production and distribution system. J. Oper. Res. Soc. 44: 637-644

Pyke D F, Cohen M A 1993 Performance characteristics of stochastic integrated productiondistribution systems. Eur. J. Oper. Res. 68: 23-48

Pyke D F, Cohen M A 1994 Multiproduct integrated production distribution systems. Eur. J. Oper. Res. 74: 18-49

Srinivasan R, Jayaraman R, Roundy R, Tayur S 1992 Procurement of common components in a stochastic environment. IBM Research Report, RC 18580

Swaminathan J M, Tayur S R 1996 Managing broader product lines through delayed differentiation using vanilla boxes. Working Paper, GSIA, Carnegie Mellon University, 1995 (revised 1996)

Treece J B. Kerwin K, Dawley H 1995 FORD: Alex Trotman's daring global strategy. Business Week (April 3): 94-104

Whitney D E 1993 Nippon Denso Co Ltd: A case study of strategic product design. Working Paper, CSDL-P 3225, Cambridge, MA-02139 